I Paroles de « pros "...

\title{
Toujours ramener le bateau au port
}

Propos recueillis par Gilles Raveneau

Anne Liardet

navigatrice

Anne Liardet est née en avril 1961 à Bourges, mais elle habite au bord de la mer depuis le plus jeune âge. Elle vit près de Brest. De son père, professeur de sculpture aux Beaux-Arts et amateur de voile, elle hérite un goût pour le bricolage qui fera merveille plus d'une fois sur ses bateaux. Passionnée de voile et de navires, elle part travailler sur un chantier naval après son baccalauréat, puis dans une voilerie pendant deux ans. Elle se retrouve ensuite à La Baule à préparer un catamaran de course avec des amis, saisit toutes les occasions pour apprendre et entre peu à peu dans le monde de la course au large. Tout en préparant des bateaux, elle navigue, fait des régates, participe à des équipages sur des multicoques, puis court sur ses propres bateaux.

En 1985, elle prend la dixième place de la Mini Transat (première femme à l'arrivée) et la première en double avec B. Audrezet. Deux ans plus tard, elle finit dixième de la Solitaire du Figaro et deuxième aux Cent Milles de Concarneau. L'année suivante, enceinte de son premier enfant, elle tente de battre le record entre New York et San Francisco via le cap Horn. En 1990, elle participe à la Route du rhum sur le multicoque CTL, alors qu'elle est enceinte de son deuxième enfant. Anne choisit alors de se consacrer à ses trois enfants, Morgan, Margot et Manon. Elle reprend le travail dans la voilerie Voiles Océans à Brest, avant de mettre le cap sur l'enseignement. Une dixaine d'années plus tard, à Brest, elle croise Catherine Chabaud, Loïc Etevenard et toute l'équipe de Kersauson. Elle se remet à rêver de navigation en solitaire autour du monde et décide de renfiler le ciré. En 2003, elle se classe neuvième du Défi Atlantique, douzième de la Transat en 2004 ; enfin, en 2005, elle s'engage dans le Vendée Globe, la prestigieuse course en solitaire autour du monde sans escale et sans assistance, dont elle prendra la onzième place.

Le vrai risque, c'est la mauvaise rencontre en mer: le cargo, les bois ou les containers qui flottent, les icebergs et éventuellement les baleines. Ton bateau peut heurter n'importe quoi, beaucoup de choses flottent sur l'eau. Tu ne peux pas le contrôler, ce sont les impondérables. Les icebergs, la nuit ou le jour dans la brume, tu ne les vois pas. Le radar est aléatoire. Parfois, tu les sens simplement à l'air froid qu'ils dégagent. Tu as aussi les « rogue waves ", qui sont des vagues démesurées par rapport à l'environnement. Elles peuvent faire trente mètres de haut. Tout à coup, tu te retrouves face à un mur. Mais ce sont des choses qui peuvent aussi t'arriver à terre. Ça n'empêche pas de vivre. Si cela doit arriver, ça arrivera. Je n'y pense pas. Mais je fais attention sur un bateau. Je regarde toujours où je mets les pieds. Tout ce que je peux contrồler, je le fais. Le reste, je l'accepte. Si tu ne l'admets pas, tu n'éprouves aucun plaisir. Naviguer est un moyen de gagner ma vie en faisant une activité que j'aime.

La meilleure solution est l'optimisme, sinon tu angoisses. Et cela ne sert à rien car il faut profiter de ton périple. C'est une activité que tu pratiques pour le plaisir, parce que tu aimes ça. Tu as des moments fugaces en mer qui sont d'une telle beauté. Tu as l'impression physique que tu fais partie de l'univers. Je ne sais pas comment l'expliquer! Tu es dans l'élément, à grande échelle. Le fait d'être seul me convient bien aussi. Ce sont des moments privilégiés.

Moi, je vais en mer pour être avec et non pas contre. Je n'y vais pas pour chercher la confrontation avec les éléments. La mer est un élément avec lequel je suis en accord. Je dis toujours que si j'étais née en montagne, j'aurais fait de l'alpinisme parce que cela correspond aux mêmes choses, aux mêmes besoins de se dépenser, d'être à l'extérieur, au théâtre de la nature... On n'imagine pas qu'en mer le spectacle soit permanent. J'ai vu des ciels que tu n'imagines même pas! Ce n'est pas le risque qui m'attire. Je n'ai pas le goût du risque. J'ai trois enfants, je n'ai pas envie. L'option que je choisis toujours est de suivre les éléments. C'est-à-dire que s'il faut perdre un peu de route, abattre ou réduire les voiles pour que le bateau passe mieux, je le fais. Tu n'es rien là-dedans. Ce n'est même pas réfléchi, c'est animal, tu le sais. Si quelqu'un me dit: "Je vais aller me battre contre les éléments », je me dis : "Il est fou. »

Parfois, il faut se battre contre soi-même aussi, c'est autre chose et pas forcément plus facile. Quand tu es vraiment fatigué, tu as envie de réduire la toile pour aller dormir trois heures. Là, tu te fais violence pour continuer. Tu as le choix : soit tu joues la course, soit tu joues ton confort. Mais tu as choisi de faire une course, tu n'es pas en croisière. En présence de concurrents, tu pousseras toujours plus loin. Tu as cette espèce d'instinct de compétition. Tu accélères tout en restant très attentif, sur le qui-vive. C'est ce que me disait Dominique Vavre ${ }^{1}$ lors du Vendée Globe. Il était passé en Atlantique, alors que j'étais encore dans le Pacifique. Il me dit au téléphone : "Toi tu as de la chance, tu es encore en bas. "Ce qui lui plaît, c'est la tension permanente dans laquelle on vit quand on est dans le Pacifique ou 
l'océan Indien. Les dangers y sont plus importants. Là, tu vis vraiment, tu es vigilant, en observation permanente. Après, sur l'Atlantique, tu te ramollis. Tu surveilles toujours tes arrières car les dépressions arrivent par l'ouest. Tu regardes le ciel en permanence. La mer y est aussi toujours très forte. Tu as des trains de dépressions qui poussent et forment la mer, cela avance vers toi et enfle. Ça monte très haut. C'est impressionnant et très beau à la fois. Tu es à cran et dans la béatitude. À la latitude du cap Horn, la mer est énorme. À un moment donné, alors que j'étais à l'intérieur du bateau et que je regardais par le hublot sous le vent, je me suis retrouvée au sommet d'une montagne d'eau. La mer n'était pourtant pas dangereuse, il y avait quarante nœuds de vent, ce qui n'est rien là-bas. J'étais en haut de cette montagne et au bord d'une pente monstrueuse. J'étais impressionnée. Et en même temps, c'était magnifique parce qu'il y avait un ciel bleu avec quelques nuages et une belle lumière... Tu n'es rien là-dedans, tu n'es vraiment rien et tu le sais.

Quand tu pousses ton bateau, tu le fais parce que tu sais qu'il va tenir, même si la limite est proche. Ton bateau parle, il te dit: "J'ai trop de toile, la quille est mal inclinée, il n'y a pas assez d'eau dans les ballasts. " Il s'exprime dans ses mouvements, dans ses manières d'aborder la mer. Tu le sens. J'ai confiance en moi sur un bateau. Je les connais. Je sais me débrouiller. Il m'est déjà arrivé de me retrouver couchée lors d'une course en solitaire, celle du Figaro. Le bateau était parti «au tas » et s'était couché sur l'eau. Dans toutes les situations de ce genre, je prends toujours cinq secondes pour observer. Je ne touche à rien et je regarde. Là, je me suis dit: «Ne sors pas comme ça, enfile d'abord ton ciré. Le bateau est couché, il ne peut pas aller plus loin. " Je ne me précipite jamais. Le pire est de paniquer. C'est là que tu fais des erreurs. Je sais que je peux contrôler le bateau, dans une certaine mesure évidemment. Si le mât tombe et que tu as de la chance, il ne perfore pas la coque et tu déclenches ta balise de secours. Si ton bateau est perforé avec une situation météo désastreuse,

tu es mal. Je ne me suis jamais trouvée dans une situation extrême comme cela.

Quand je pars en mer, je ne suis pas aiguillonnée par une pointe de peur, mais plutôt par le fait de me retrouver seule. Je le fais malgré le risque. Je me contente de dominer mon sujet le mieux possible. Le bateau ne doit pas être plus fort que toi. Tu dois anticiper. C'est une manière de réduire le risque, car il est lié à la "casse" du bateau. Si tu focalises sur l'accident qui peut arriver, c'est là que tu risques de faire des erreurs. Si tu y penses trop, tu n'es plus assez attentif à l'environnement. Tu peux prévenir les dégâts matériels en réduisant la voile suffisamment tôt, plutôt que de "rentrer dans le grain $»^{2}$ par exemple.
Une de mes satisfactions est d'avoir toujours ramené mon bateau. Un rapport se crée forcément entre toi et lui. Tu vis des choses fortes avec lui. Ce n'est pas une personne, bien sûr, mais c'est ton compagnon de route. C'est très important pour moi de revenir à deux. On part à deux, on revient à deux. Perdre un bateau serait un vrai coup dur. Sur la course Jacques Vabre, sur dix multicoques au départ, quatre seulement sont rentrés! Perdre son bateau, c'est pire qu'arriver dernier.

Je ne navigue pas pour prendre des risques. Mais je le fais aussi parce que ce n'est pas "bordé " comme peut l'être la vie à terre, où tout est balisé. Tu prends ta bouffée d'espace et de liberté. Le risque sportif est valorisé parce qu'il y a un public que cela fait rêver. C'est paradoxal parce que les gens en rêvent et on les assomme de réglementations et d'assurances. La recherche de sécurité aujourd'hui est épouvantable. On nous apprend à rentrer dans un moule et quand le moule se fend, tu fais comment? On nous endort. On en perd l'instinct de survie, la capacité à se protéger soi-même et à sentir les dangers. Les gens s'en remettent aux autres et ne se prennent plus en charge. C'est un moyen de contrôler le peuple aussi. Si tu as peur de sortir, tu restes chez toi. Si tu as peur d'aller en mer parce qu'il y a des dangers, idem. Tu es "bordé ". Si tu n'acceptes pas un minimum de risque, tu ne fais plus rien. Ce qui me terrifie, ce sont les victimes d'accident qui cherchent absolument un responsable. Il n'est plus possible aujourd'hui d'accepter la malchance. Personne n'est infaillible. Ce n'est pas en attaquant le pseudo-responsable en justice qu'un drame est réparé.

Dans les sports, tu es libre de prendre les risques que tu veux ou presque. C'est un espace qui n'est pas aussi encadré que la vie sociale en général. On te demande bien entendu d'emporter sur un bateau tel ou tel matériel de sécurité. Parfois, tu rigoles lorsqu'il s'agit d'instruments ne pouvant être mis en œuvre qu'à plusieurs, comme l'appareil de recherche d'hommes à la mer, et que tu es seul. Quand tu fais le compte de ce que tu devrais avoir sur le dos à chaque fois que tu sors sur le pont, tu es comme un scaphandrier. Tu ne peux plus bouger. Je préfere regarder où je pose les pieds. Si tu tombes à l'eau dans le Pacifique sud, malgré ta balise, en dix minutes tu es mort car l'eau est tellement froide que tu ne résistes pas. Tu ne te balades pas tout le temps avec ta combinaison de survie. Ce sont les assureurs et les organisateurs de course que cela tranquillise.

\section{Notes}

\footnotetext{
1. Navigateur, concurrent du dernier Vendée Globe 2005.

2. Cette expression signifie naviguer dans la tempête, le mauvais temps.
} 